\section{In Situ Investigations of Structure- activity correlations of Mixed Molybdenum Oxide Catalysts}

\author{
E. Rödel, R.E. Jentoft, T. Ressler*
}

Abteilung Anorganische Chemie, Fritz-Haber-Institut der MaxPlanck-Gesellschaft, Faradayweg 4-6, D-14195 Berlin

Keywords: In situ; XAS; XRD; (Mo,V,W) Mixed oxide catalyst

\section{Introduction}

Molybdenum containing catalysts are widely used for the selective oxidation of propene in the presence of gas phase oxygen at temperatures above $\sim 600 \mathrm{~K}$. Crystalline $(\mathrm{Mo}, \mathrm{V}, \mathrm{W})_{5} \mathrm{O}_{14}$ constitutes a three-dimensional model system for the more complex molybdenum based mixed oxide catalysts that are employed industrially for various partial oxidation reactions [1]. However, little is known about the cooperation of the different cations and their role for the stabilization of the active phase. Here we present investigations of the structural evolution of $(\mathrm{Mo}, \mathrm{V}, \mathrm{W})_{5} \mathrm{O}_{14}$ under propene oxidation conditions at different temperatures using in situ X-ray absorption spectroscopy (XAS) and in situ X-ray diffraction (XRD).

\section{Experimental}

The $\mathrm{Mo}_{0.68} \mathrm{~V}_{0.23} \mathrm{~W}_{0.09}$ oxide catalyst was prepared by spray-drying mixed solutions of ammonium heptamolybdate, ammonium metatungstate and vanadyl oxalate and calcined at $623 \mathrm{~K}$ in air and $713 \mathrm{~K}$ in helium [2]. Combined in situ XRD/MS experiments were performed on a STOE STADI P powder diffractometer employing a Bühler HDK S1 chamber. The gas phase composition at the cell outlet was analyzed on line with a mass spectrometer. Measurements were conducted under atmospheric pressure in flowing reactants. Combined transmission XAS/MS experiments were performed at the Mo-K edge at beamline X1 at HASYLAB in a flowreactor at atmospheric pressure in flowing reactants $(\sim 30 \mathrm{ml} / \mathrm{min})$. Time-resolved in situ XAS experiments were carried out at the Mo$\mathrm{K}$ edge utilizing an energy-dispersive spectrometer (ESRF, ID24).

\section{Results}

The $(\mathrm{Mo}, \mathrm{V}, \mathrm{W})_{5} \mathrm{O}_{14}$ material prepared exhibited an onset of catalytic activity at about the same temperature as $\mathrm{MoO}_{3}(\sim 600 \mathrm{~K})$ indicating similar active sites and structure-activity correlations. Isothermal in situ XAS experiments while changing the gas atmosphere from propene to propene and oxygen revealed a decreasing average Mo oxidation state during catalysis with increasing reaction temperature. In situ XRD showed that the catalyst exhibits no phase changes during catalysis under mildly reducing conditions ( $10 \%$ propene, $10 \%$ oxygen). The $(\mathrm{Mo}, \mathrm{V}, \mathrm{W})_{5} \mathrm{O}_{14}$ employed is stable in oxygen up to $773 \mathrm{~K}$, whereas it is strongly reduced in $10 \%$ propene or $10 \% \mathrm{H}_{2}$ at temperatures above $723 \mathrm{~K}$.

[1] R. K. Grasselli, Catalysis Today 1999, 49, 141.

[2] S. Knobl et al., J. Catalysis 2003, 215, 177.

\section{Tieftemperaturuntersuchungen an synthetischem $\mathrm{Cu}_{3} \mathrm{BiS}_{3}$}

\author{
A. Roduch, A. Pfitzner* \\ Institut für Anorganische Chemie, Universität Regensburg, \\ Universitätsstraße 31, D-93040 Regensburg
}

Keywords: $\mathrm{Cu}_{3} \mathrm{BiS}_{3}$ (Wittichenit); Low temperature investigations; Phase transitions.

Von $\mathrm{Cu}_{3} \mathrm{BiS}_{3}$ kennt man bis jetzt drei Modifikationen. Es ist bei Raumtemperatur orthorhombisch mit $a=7.723 \AA$, $b=10.395 \AA$ und $c=6.716 \AA$, RG $P 2_{1} 2_{1} 2_{1}$ [1] und bei $T>190^{\circ} \mathrm{C}$ orthorhombisch mit $a=7.705 \AA, b=10.400 \AA, c=6.720 \AA$, RG Pnma [2]. Für $110^{\circ} \mathrm{C}<T<190^{\circ} \mathrm{C}$ wird ein inkommensurables, orthorhombisches Intermediat beobachtet mit Satelliten entlang $c^{*}[2,3]$. In allen Strukturen liegen diskrete $\mathrm{BiS}_{3}{ }^{3-}$-Einheiten vor, deren Anordnung nahezu gleich bleibt. Die einzelnen Modifikationen unterscheiden sich in der Verteilung der trigonal planar koordinierten Kupferatome.

Mizota et al. [4] berichten über eine Phasenumwandlung bei $-38^{\circ} \mathrm{C}$, die wir genauer untersucht haben. Röntgenpulveruntersuchungen bei $-100^{\circ} \mathrm{C}$ ergeben eine orthorhombische Metrik mit $a=7.729(2) \AA, b=10.280(2) \AA, c=6.677(1) \AA$. Die Struktur konnte durch Röntgenbeugung an einem Einkristall bei $-130^{\circ} \mathrm{C}$ ermittelt werden. Es zeigt sich, dass beim Übergang von der Raumzur Tieftemperaturmodifikation eine Erhöhung der Symmetrie von $P 2_{1} 2_{1} 2_{1}$ nach $P n m 2_{1}$ eintritt. Die Anordnung der $\mathrm{BiS}_{3}{ }^{3-}$-Einheiten bleibt dabei erhalten. Eine Änderung zeigt sich in der Verteilung des Kupfers und der Koordination des Schwefels. Während bei Raumtemperatur ausschließlich $\left[\mathrm{SBiCu}_{3}\right]$-Einheiten vorliegen, existieren bei tiefen Temperaturen $\left[\mathrm{SBiCu}_{3}\right]-, \quad\left[\mathrm{SBiCu}_{2}\right]-$ und $\left[\mathrm{SBiCu}_{4}\right]$-Baugruppen.

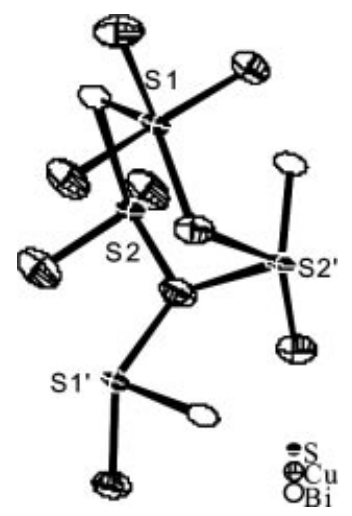

Abb. 1 Koordination der Schwefelatome mit Bismut und Kupfer in $\mathrm{Cu}_{3} \mathrm{BiS}_{3}$ bei $-130^{\circ} \mathrm{C}$.

[1] V. Kocman, E. Nuffield, Acta Crystallogr. 1973, B29, 2528.

[2] E. Makovicky, J. Solid State Chem. 1983, 49, 85.

[3] E. Makovicky, N. Jb. Miner. Abh. 1992, 168, 185.

[4] T. Mizota, A. Inoue, Y. Yamada, A. Nakatsuka, N. Nakayama, Mineral. J. 1998 , 20, 81.

DOI: 10.1002/zaac.200470126 